\title{
Interfacial erosion: A three-dimensional numerical model
}

\author{
Frédéric Golay ${ }^{\mathrm{a}, \mathrm{b}}$, Damien Lachouette ${ }^{\mathrm{a}, \mathrm{b}}$, Stéphane Bonelli ${ }^{\mathrm{b}}$, Pierre Seppecher ${ }^{\mathrm{a}, *}$ \\ a IMATH, université du Sud Toulon-Var, avenue de l'université, 83957 La Garde, France \\ ${ }^{\mathrm{b}}$ Unité ouvrages hydrauliques et hydrologie, Cemagref Aix-en-Provence, 3275 route de Cézanne CS 40061, 13182 Aix-en-Provence cedex 5, France
}

\section{A R T I C L E I N F O}

\section{Article history:}

Received 4 March 2010

Accepted after revision 1 June 2010

Available online 17 June 2010

\section{Keywords:}

Soils

Erosion

Finite volume

Penalization

Level set

Interface

Fictitious domain

\begin{abstract}
A B S T R A C T
The aim of this study was to develop a numerical model to simulate the surface erosion occurring at a fluid/soil interface subject to a flow process. We used a penalization procedure to compute flow around obstacles. A fictitious domain method allowed the use of fast and efficient finite volumes approximations on Cartesian meshes and avoided unstructured body-fitted meshes. The water/soil interface evolution was described with a Level Set function. Several numerical simulations confirmed the model's ability to predict the interfacial erosion of soils.
\end{abstract}

(C) 2010 Académie des sciences. Published by Elsevier Masson SAS. All rights reserved.

\section{Introduction}

The present study deals with the internal erosion process subject to tangential water flow. The present approach differs from previous theories in that our description deals with a sharp fluid/soil interface. The basic balance equations and erosion constitutive law were developed by Brivois et al. [1] and Bonelli and Brivois [2]. For the sake of simplification, sedimentation and deposition processes are neglected here, and the flow is assumed to be dilute, Lachouette et al. [3]. A penalization procedure, incorporating a fictitious domains method, Angot et al. [4], is used to compute the flow around obstacles. The evolution of the water/soil interface is described with a Level Set function. Spatial derivatives are computed with an order 5 WENO (weighted essentially non-oscillatory) scheme, while time derivatives are computed with a fourth order (RK4) Runge-Kutta scheme. A finite volume formulation is developed on a Cartesian MAC mesh.

\section{Governing equations}

Here, we focus on the surface erosion under tangential flow of cohesive soils (like clay), considered as impervious materials. Our description deals with singular (or discontinuous) fluid/soil interfaces. As the erosion process is much slower than the fluid flow and as the eroded particles are small $(10-100 \mu \mathrm{m})$, the fluid flow is assumed to be a dilute flow and we neglect the variation of the fluid density and redeposition. These hypotheses were validated in our previous experimental tests (such as the "Hole Erosion Test") (Bonelli and Brivois [2]). The fluid flow is described by an incompressible Navier-Stokes system of equations:

$$
\nabla \cdot \mathbf{u}=0
$$

\footnotetext{
* Corresponding author.

E-mail addresses: frederic.golay@univ-tln.fr (F. Golay), stephane.bonelli@aix.cemagref.fr (S. Bonelli), seppecher@imath.fr (P. Seppecher).
} 


$$
\rho \frac{\partial \mathbf{u}}{\partial t}+\rho(\mathbf{u} \cdot \nabla) \mathbf{u}-\nabla \cdot\left(\mu\left(\nabla \mathbf{u}+\nabla^{\mathrm{T}} \mathbf{u}\right)\right)+\nabla p=0
$$

Here, $\mathbf{u}$ denotes the vector velocity field, $\rho$ the fluid density, $\mu$ the viscosity and $p$ the pressure. The mass and momentum conservation (Eqs. (1) and (2)) are valid in the fluid domain $\Omega_{f}$. A part of its boundary, the interface between the fluid and the soil $\Gamma$, is in movement because of the erosion process. Let us denote $\mathbf{c}_{\Gamma}$ the celerity of the interface $\Gamma$ and $\mathbf{n}$ its unit normal vector oriented from the soil to the fluid. At the interface the mass conservation implies a jump condition:

$$
\llbracket \rho\left(\mathbf{c}_{\Gamma}-\mathbf{u}\right) \cdot \mathbf{n} \rrbracket=0
$$

As we consider surface erosion between an impervious soil $(\mathbf{u}=0)$ and a tangential fluid flow $(\mathbf{u} \cdot \mathbf{n}=0)$, the mass flux crossing the interface and evacuated by the dilute flow (of density $\rho$ ) is defined as follows:

$$
\dot{m}=\rho \mathbf{c}_{\Gamma} \cdot \mathbf{n}
$$

It is then necessary to describe this erosion process. For our experiments and in line with Brivois et al. [1] and others (Foster and Fell [5], Wan and Fell [6]), we use a constitutive erosion law driven by tangential shear stress, written in the form of a threshold law as follows:

$$
\dot{m}= \begin{cases}k_{e r}\left(|\tau|-\tau_{c}\right) & \text { if }|\tau|>\tau_{c} \\ 0 & \text { otherwise }\end{cases}
$$

where $\tau$ is the tangential (shear) stress exerted on the soil, while $k_{e r}$ and $\tau_{c}$ are positive parameters of the erosion law.

\section{Mathematical model}

We use a spatial discretization which is not boundary-fitted to the fluid domain. The main idea consists in incorporating the original domain of study in a geometrically bigger and simpler one called "fictitious domain". This "penalized method", is popular because it allows the use of fairly structured meshes. The fictitious domain method is now widely used, for example, in fluid-porous-solid flows and has been mathematically justified (see e.g. Angot [7], Angot et al. [4], Diaz-Goano et al. [8], Khadra et al. [9]). Let us take a computation domain $\Omega$ (fictitious domain) with the previous fluid domain $\Omega_{f}$ and the soil domain $\Omega_{s}$. The penalized method allows describing the behavior of the two sub-domains with a set of equations defined in the whole domain introducing a new term in the balance equation (2):

$$
\rho \frac{\partial \mathbf{u}}{\partial t}+\rho(\mathbf{u} . \nabla) \mathbf{u}-\nabla \cdot\left(\mu\left(\nabla \mathbf{u}+\nabla^{\mathrm{T}} \mathbf{u}\right)\right)=-\nabla p-H \frac{\mu}{K_{s}} \mathbf{u}
$$

where $K_{S}$ is a penalization coefficient (a very small number having the dimension of a permeability) and $H$ is the characteristic function of the soil domain, which is unity within $\Omega_{s}$ and zero elsewhere. As the last term of the right-hand side of (6) is zero in the fluid domain, it corresponds to the Navier-Stokes equation. In the soil domain, penalization leads to very low values of $\mathbf{u}$, and the left-hand side of (6) is negligible, as well as the viscous part of the stress tensor.

As we consider a sharp interface, the Level Set method is an appropriate formulation for describing the interface. It was introduced by Osher and Sethian [10] and is widely used in multi-fluid flows (e.g. Chang et al. [11], Olson and Kreiss [12], Sussman et al. [13]) and the fictitious domain approach (e.g. Chantalat et al. [14]). We introduce a function $\psi$, that is negative in the fluid domain $\Omega_{f}$ and positive in the soil domain $\Omega_{s}$. Interface $\Gamma$ is represented by the zero level set of $\psi$, and the value of $H$ depends only on $\psi$. A relevant approach is to choose this function as the signed distance to the interface. The displacement of the interface is then driven by a transport equation:

$$
\frac{\partial \psi}{\partial t}+\mathbf{c} . \nabla \psi=0
$$

where $\mathbf{c}$ is a suitable extension on the whole domain $\Omega$ of the celerity of the interface $\mathbf{c}_{\Gamma}$ defined in (4), which is defined only on the interface. Note that the normal to the interface is given by the gradient of the Level Set function.

\section{Numerical modeling}

As the erosion process is much slower than the main fluid flow, these two phenomena are split, by using different time scales. For the fluid flow (1), (6), we use a finite volume method on a staggered grid for spatial discretization. This method, known as the Marker and Cell (MAC) method formulated by Harlow and Welch [15], is now widely used in many applications (see, for example, Patil and Tiwari [16]). The time derivative is approached with an explicit Euler scheme. In order to deal with the constraint of divergence-free velocity, we use the augmented Lagrangian method (see e.g. Vincent and Caltagirone [17], Galusinski and Vigneaux [18]). This iterative predictor/corrector method consists in solving an optimization problem by solving a velocity-pressure saddle point with an Uzawa algorithm, in order to converge towards a solution which satisfies the incompressibility constraint. The linear algebra system is solved numerically with an iterative Bi-Conjugate Gradient Stabilized algorithm (BiCGSTAB). This algorithm is particularly effective for managing computer memory, because it does away with the need to build and store the linear matrix. We simply need to express the product of the matrix 


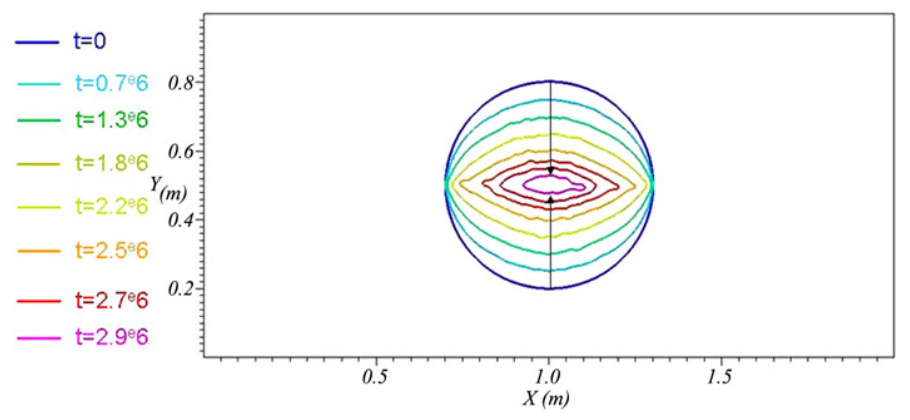

Fig. 1. Evolution of the shape of the cylinder through time.

and the vector of the unknowns. In (7), which describes the interface motion, the spatial gradients are approached with a high order Weighted Essentially Non-Oscillating (WENO5) scheme introduced by Jiang and Peng [19], while the time discretization uses a fourth order Runge-Kutta scheme (RK4).

The computation of the shear stress at the interface is crucial because it determines the rate of the erosion (5). The numerical estimation of the velocity gradient can be difficult because it is discontinuous across the interface. As the velocity almost vanishes in the soil, we obtain an accurate estimation of the shear stress by computing it with the fluid velocity only (on the fluid side on the interface), i.e. by extending this velocity in a few cells in the soil near the interface, in a way similar to the "ghost-fluid" method (Fedkiw [20]). To improve accuracy we propose a better approximation of the shear stress at the interface. Indeed the velocity gradient is computed at the center $\mathbf{x}$ of the cell and not at the interface. That is why we extrapolate the gradient (or the stress) to the interface:

$$
\tau(\mathbf{x}-\psi \mathbf{n}) \approx \tau(\mathbf{x})-\psi \mathbf{n} . \nabla \tau
$$

Recall that $\psi$ denotes the distance to the interface in (8).

The different parts of the numerical implementation have been validated. Among other tests, the flow solver has been tested on a Couette flow; the transport equation has been validated by the Zalesak's disk test; the erosion process has been successfully compared in the case of the "Hole Erosion Test" to the analytical solution developed by Bonelli and Brivois [2].

\section{Results}

\subsection{Erosion of a soil cylinder}

We considered a test case: the erosion of a fixed cylinder of soil (radius $0.3 \mathrm{~m}$, length $2 \mathrm{~m}$, height $1 \mathrm{~m}$, mesh $100 \times 50$ ) in a channel.

The fluid flow was approached by a stationary Stokes model. We imposed a pressure gradient from the left to the right and a wall condition at the top and the bottom of the computational domain $\left(\rho_{f}=1000 \mathrm{~kg} / \mathrm{m}^{3}, \mu_{f}=0.001 \mathrm{~Pa} / \mathrm{s}\right.$, $\left.k_{e r}=0.001 \mathrm{~s} / \mathrm{m}, \tau_{c}=10-20 \mathrm{~Pa}, r_{1}=r_{2}=1, c f l=0.8, \delta P=0.1 \mathrm{~Pa}\right)$.

Fig. 1 shows the evolution of the cylinder due to interfacial erosion. We then verified that the shape and flow were symmetrical, as was expected, and that the cylinder was mostly eroded at the upper and lower part of the interface. A stationary Stokes model was used because the erosion process is much slower than the flow process, as highlighted by the erosion characteristic time (Bonelli and Brivois [2]). A parametric study showed that the flow can be assumed to be stationary until the erosion coefficient reaches a low value $\left(k_{e r} \leqslant 10^{-3}\right)$. A parametric study of the mesh underlined the consistency of the formulation.

\subsection{Erosion around a bridge}

In order to test several three-dimensional computations, we consider the erosion of soil around a bridge pier (Figs. 2 and 3). A wall condition is applied at the bottom of the computational box, a symmetric condition at the top, a periodic condition on the sides and a pressure gradient is imposed between the inlet and the outlet. We use a coarse mesh $150 \times$ $50 \times 50$ and a Stokes solver. In a realistic case, a free surface should be considered (e.g. Golay and Helluy [21]) and the full Navier-Stokes equation should be used. These developments are out of the scope of this study: here we only intend to check the possibility of simulating an erosion process in a 3D case.

Two Level Set functions were used: one for the interface with the bridge pier, which was a non-erodible material, and another for the soil-fluid interface. The erosion process stopped as the shear stress at the interface reached the threshold shear stress. This numerical simulation required about 2 days on a personal computer (Xeon $3 \mathrm{GHz}$ ), so it proved the feasibility of such computations and thus more realistic ones. 


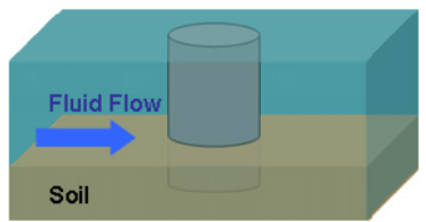

Fig. 2. Erosion around a bridge pier.
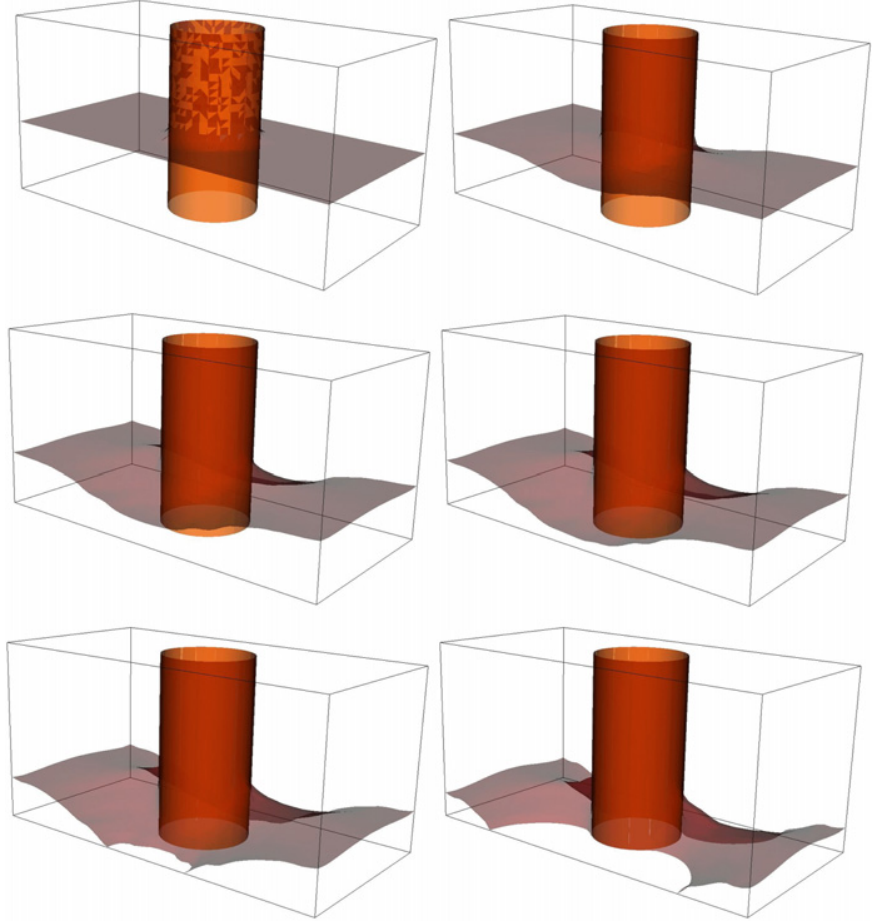

Fig. 3. Erosion around a bridge pier at $t=0,1.9 \times 10^{7}, 4.2 \times 10^{7}, 5.5 \times 10^{7}, 6.6 \times 10^{7}, 9.5 \times 10^{7}$.

\section{Conclusion}

The aim of this study was to draw up a numerical model for simulating interfacial erosion under a tangential flow. We presented an erosion model with a unified model using a penalization procedure (fictitious domain method), thereby avoiding prohibitive remeshing methods. The water/soil interface evolution was described with a Level Set function. A finite volume formulation was developed classically on a Cartesian MAC mesh to approximate the flow, in order to implement appropriate fast solvers. The ability of the model to predict the interfacial erosion of soils was confirmed by presenting several three-dimensional simulations. In further works, we intend to improve the fluid solver, for example with turbulent models or parallel formulations, in order to achieve more realistic simulations. For turbulent flows, the challenge will be to give a sufficiently accurate description of the boundary layers in order to get correct values of the shear stress and consequently of the erosion rate.

\section{Acknowledgements}

This work was funded by the French National Research Agency (ANR) through the COSINUS program (CARPEINTER project $\mathrm{n}^{\circ}$ ANR-08-COSI-002), and by the Région Provence Alpes Côte d'Azur.

\section{References}

[1] O. Brivois, S. Bonelli, R. Borghi, Soil erosion in the boundary layer flow along a slope: A theoretical study, Eur. J. Mech. B Fluids 26 (2007) 707-719.

[2] S. Bonelli, O. Brivois, The scaling law in the hole erosion test with a constant pressure drop, Internat. J. Numer. Methods Engrg. 32 (2008) $1573-1595$.

[3] D. Lachouette, F. Golay, S. Bonelli, One-dimensional modeling of piping flow erosion, C. R. Mecanique 336 (2008) $731-736$.

[4] P. Angot, C.-H. Bruneau, P. Fabrie, A penalization method to take into account obstacles in incompressible viscous flows, Numer. Math. 81 (1999) 497-520.

[5] M. Foster, R. Fell, Assessing embankment dam filters that do not satisfy design criteria, J. Geotech. Geoenviron. Eng. 125 (7) (2001) $398-407$. 
[6] C. Wan, R. Fell, Investigation of rate of erosion of soils in embankment dams, J. Geotech. Geoenviron. Eng. 130 (4) (2004) $373-380$.

[7] P. Angot, Analysis of singular perturbations on the Brinkman problem for fictitious domain models of viscous flows, Math. Methods Appl. Sci. 22 (1999) 1395-1412.

[8] C. Diaz-Goano, P.D. Minev, K. Nandakumar, A fictitious domain/finite element method for particulate flows, J. Comput. Phys. 192 (2003) $105-123$.

[9] K. Khadra, P. Angot, S. Parneix, J.P. Caltagirone, Fictitious domain approach for numerical modelling of Navier-Stokes equations, Internat. J. Numer. Methods Fluids 34 (2000) 651-684.

[10] S. Osher, J. Sethian, Fronts propagating with curvature dependent speed: Algorithm for tracking material interface, J. Comput. Phys. 39 (1981) $201-225$.

[11] Y.C. Chang, T.Y. Hou, B. Merriman, S. Osher, A level set formulation of Eulerian interface capturing methods for incompressible fluid flows, J. Comput. Phys. 124 (1996) 449-464.

[12] E. Olson, G. Kreiss, A conservative level set method for two phase flow, J. Comput. Phys. 210 (2005) $225-246$.

[13] M. Sussman, P. Smereka, S. Osher, A level set approach for computing solutions to incompressible two-phase flows, J. Comput. Phys. 114 (1994) 146-159.

[14] F. Chantalat, C.H. Bruneau, C. Galusinski, A. Iollo, Level-set, penalization and Cartesian meshes: A paradigm for inverse problems and optimal design, J. Comput. Phys. 228 (2009) 6291-6315.

[15] F.H. Harlow, J.E. Welsh, Numerical calculation of time dependent viscous incompressible flow with free surface, Phys. Fluids 8 (12) (1965) 2182-2189.

[16] P.P. Patil, S. Tiwari, Computation of flow past complex geometries using MAC algorithm on body-fitted coordinates, Eng. Appl. Comput. Fluids Mech. 3 (1) (2009) 15-27.

[17] S. Vincent, J.P. Caltagirone, A one-cell local multigrid method for solving unsteady incompressible multiphase flows, J. Comput. Phys. 163 (2000) 172215.

[18] C. Galusinski, P. Vigneaux, On stability condition for bifluid flows with surface tension: Application to microfluidics, J. Comput. Phys. 227 (2008) 6140-6164.

[19] G.S. Jiang, D. Peng, Weighted ENO schemes for Hamilton-Jacobi equations, J. Sci. Comput. 21 (6) (2000) $2126-2143$.

[20] R. Fedkiw, Coupling an Eulerian fluid calculation to a Lagrangian solid calculation with the ghost fluid method, J. Comput. Phys. 175 (2002) $200-224$.

[21] F. Golay, P. Helluy, Numerical schemes for low Mach wave breaking, Int. J. Comput. Fluid Dyn. 21 (2) (2007) 69-86. 